\title{
Calculations of the Effective Thermal Conductivity in a Model of Syntactic Metallic Hollow Sphere Structures Using a Lattice Monte Carlo method
}

\author{
T. Fiedler, a , A. Öchsner, ${ }^{2, \text { b }, ~ I . V . ~ B e l o v a ~}{ }^{3, \mathrm{c}}$ and G.E. Murch ${ }^{3, \mathrm{~d}}$ \\ ${ }^{1}$ Department of Mechanical Engineering, University of Aveiro, 3810-193 Aveiro, Portugal \\ ${ }^{2}$ Department of Applied Mechanics, Faculty of Mechanical Engineering, Technical \\ University of Malaysia, 81310 UTM Skudai, Johor, Malaysia \\ ${ }^{3}$ Diffusion in Solids Group, Centre for Geotechnical and Materials Modelling, \\ School of Engineering, \\ The University of Newcastle, Callaghan, NSW 2308, Australia \\ a TFiedler@mec.ua.pt, b Oechsner@fkm.utm.my \\ c Irina.Belova@newcastle.edu.au, d Graeme.Murch@newcastle.edu.au
}

Keywords: Thermal conductivity, MHSS, Lattice Monte Carlo

\begin{abstract}
In this paper, a Lattice Monte Carlo method is used to determine the effective thermal conductivity in two dimensional models of adhesively bonded metallic hollow sphere structures (MHSS). In contrast to earlier approaches, more realistic distributions of spheres without the simplification of cubic symmetric arrangements are considered in this study. For the Monte Carlo analyses, two-dimensional periodic lattices representing different cutting planes through MHSS are generated. Therefore, an algorithm is used which sequentially fills the lattice by adding cut spherical shells and inclusions in the matrix. Another focus of this work is the analysis of the influence of different geometric circle distributions on the effective thermal conductivity. The findings of the random arrangements are also compared to a regular primitive cubic arrangement and with a Maxwell-type approach.
\end{abstract}

\section{Introduction}

Porous metals are characterised by high specific stiffness, the ability to absorb high amounts of energy and the potential for noise control, mechanical damping and thermal insulation 0 . However, classical cellular metals such as aluminium metallic foams often exhibit inconstant material parameters 0 due to their stochastic geometry. Local density inhomogeneities [3,4] yield a scattering of macroscopic properties. This problem can be decreased by metallic hollow sphere structures (MHSS) which are assembled by spheres with a defined geometry and possess a more homogeneous structure which results in optimized properties. MHSS exhibit a low thermal conductivity in comparison to their metallic sphere wall materials. In particular, adhesively bonded MHSS show very low thermal conductivities, due to the insulating effect of the adhesive matrix between the metallic shells of the spheres. Consequently, MHSS are of interest as thermal insulators. 
a)

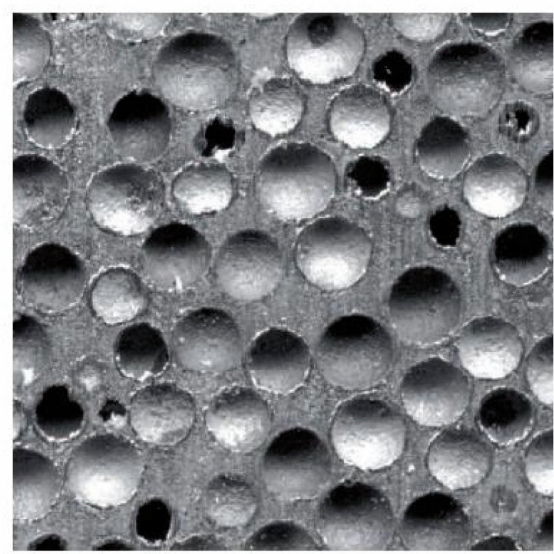

b)

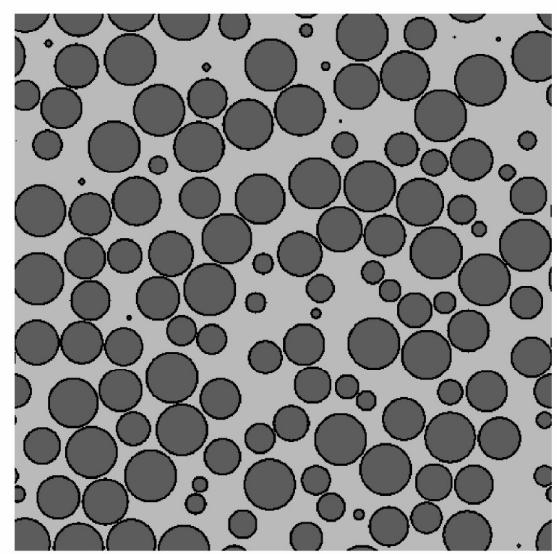

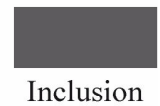

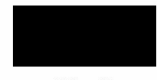

Shell

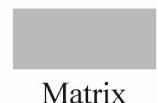

Matrix

Fig. 1 Cross section of MHSS: a) Experimental specimen, b) A typical distribution used in the Monte Carlo calculations.

Earlier research on the thermal properties of cellular metals mainly focused on open-celled structures (e.g. [5,6]). Due to their interconnected porosity, open-celled metal foams can be used in heat exchangers [7,8] or resistance heaters [9]. The investigated MHSS exhibit no interconnected porosity and therefore belong to the group of closed-cell structures. Lu and Chen [10] investigated the thermal transport and fire inhibiting properties of closed-cell aluminium alloys. They found a strong dependence of the thermal conductivity of the cellular metals on the cell shape, connectivity and topology. Furthermore, they noted a decrease of the thermal conductivity in the presence of geometrical imperfections. Baumeister and colleagues [11] investigated the thermal properties of syntactic hollow sphere composites. Corundum based hollow spheres were embedded in an epoxy matrix and the thermal expansion coefficient was determined. It was found that the thermal behaviour of these composites was mainly governed by the epoxy resin used. The effective thermal conductivity of adhesively bonded and sintered MHSS was numerically investigated in $[12,13]$. Within the scope of these finite element analyses, a strong dependence of the thermal properties on the joining technology and morphology of the structures was found. In [14], finite element and experimental analysis of the effective thermal conductivity of adhesively bonded MHSS were conducted.

Another method has recently been developed that is a particularly flexible one for addressing complex phenomenological mass and thermal diffusion problems. It is based on the lattice model and is addressed using Monte Carlo methods where it is often called the Lattice Monte Carlo method, see the recent review [15]. Although Monte Carlo methods traditionally have gained the reputation for being especially demanding of computer time, this is generally no longer a major consideration in their implementation: contemporary PCs can readily and accurately cope with the computational demands often in less than several hours computational time. In the present study, the Lattice Monte Carlo method is used to determine the effective conductivity in a model of MHSS.

\section{Geometry}

Metallic hollow sphere structures are assembled by single hollow spheres which can be manufactured by a sintering process [16]. Various joining technologies such as sintering, soldering and adhering can be used to join the single hollow spheres to interdependent structures [17]. Adhesion is the most economic way of joining and therefore is attractive for a wide range of potential applications. Syntactic MHSS are manufactured by pouring single hollow spheres into a mould and adding an epoxy resin to the fill. Figure 1 a) shows the cross section of a syntactic MHSS specimen. The outer radii $R$ of the metallic hollow spheres are $1.5 \mathrm{~mm}$ and the sphere wall thicknesses are $0.075 \mathrm{~mm}$. The radii $r_{i}$ (c.f. Fig. 2) of their circular cross sections depend on the distance $h_{i}$ of their centre points to the cutting plane and are given by the relation $r_{i}\left(h_{i}\right)=\sqrt{R^{2}-h_{i}^{2}}$. 
Figure $1 \mathrm{~b}$ ) shows an example of a two-dimensional periodic model which is used in the Monte Carlo calculation. The models were generated using an algorithm which sequentially fills spheres into a lattice at randomly selected coordinates $\mathrm{C}_{\mathrm{i}}\left(X_{i}, Y_{i}, h_{i}\right)$. Thereby, the distance $h_{i}$ must be smaller than the sphere radius $R$ in order to ensure intersection with the cutting plane. A further random number defines the position $p_{i}$ of the centre point $\mathrm{C}_{i}$ relative to the cutting plane (below: $p_{i}=1$, above: $p_{i}=-1$ ). In the next step of this algorithm, intersection of the last added sphere with preexisting spheres is investigated. Therefore, the distances $\left(d_{i}\right)$ between the spheres were calculated:

$$
d_{i}=\sqrt{\left(X_{n}-X_{i}\right)^{2}+\left(Y_{n}-Y_{i}\right)^{2}+\left(p_{n} \cdot h_{n}-p_{i} \cdot h_{i}\right)^{2}} \cdot \quad(i=1 \ldots n-1)
$$

If any distance $d_{i}$ is smaller than two times the sphere radius $R$, at least two spheres are intersecting and the last added sphere $n$ must be removed. Figure 2 displays this condition for the example $h_{i}=$ 0 . Spheres are added in the model until $10^{7}$ repeated attempts fail due to intersection. In this case, it is assumed that no further spheres can be positioned in the gaps between the previously added spheres. In order to analyse a representative cross section of the MHSS, each lattice contains at least 100 circles.

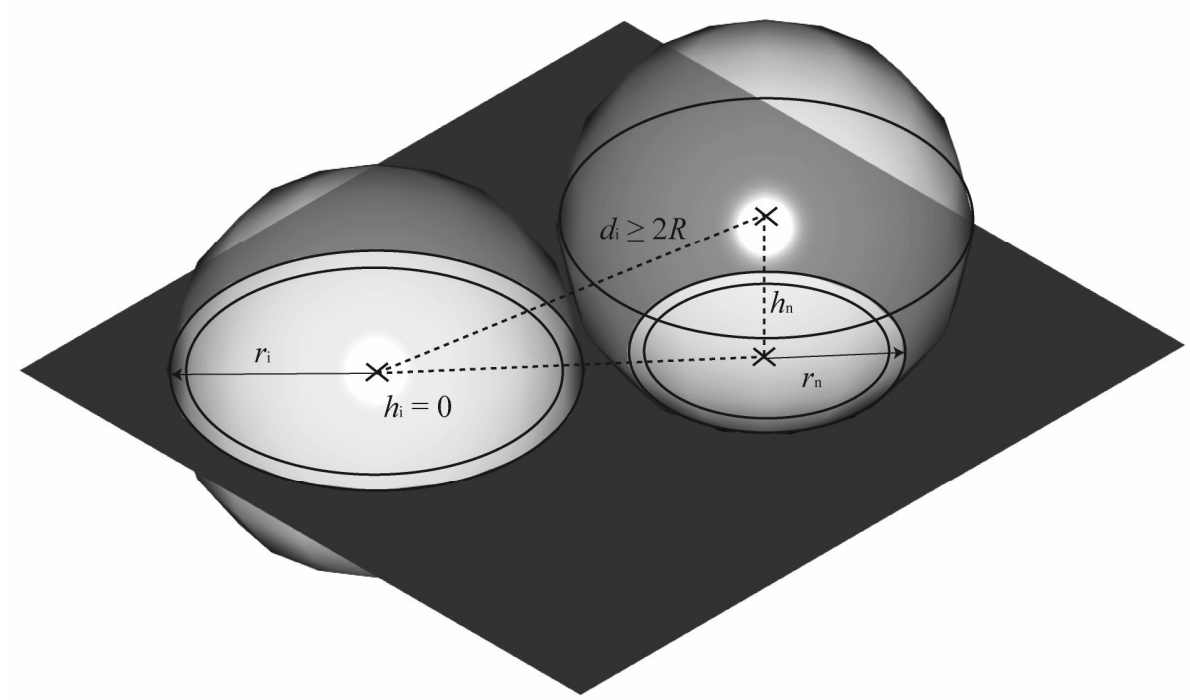

Fig. 2 Adding of circles in the cutting plane.

In the final step of the lattice generation, two dimensional matrices $\mathbf{M}(X, Y)$ with a resolution $500 \mathrm{x}$ 500 are created. A particular cross section through a MHSS can be characterised by its area fractions: the sum of the area fraction of the matrix $A_{\mathrm{M}}$, the shell $A_{\mathrm{S}}$ and the inclusion $A_{\mathrm{I}}$ is equal to unity. The area fractions of the lattice models are simply obtained by summing up the lattice points related to each phase and dividing them by the total number of lattice points $\left(500^{2}\right)$. The average density of the MHSS can then be calculated:

$$
\bar{\rho}=A_{\mathrm{S}} \cdot \rho_{\mathrm{S}}+A_{M} \cdot \rho_{\mathrm{M}},
$$

where $\rho_{\mathrm{M}}=1.13 \mathrm{~g} / \mathrm{cm}^{3}$ is the density of the epoxy matrix and $\rho_{\mathrm{S}}=6.2 \mathrm{~g} / \mathrm{cm}^{3}$ (both values were measured at the University of Aveiro, Portugal) is the density of the metallic sphere wall material. Table 1 shows the area fractions and average densities of the lattices employed. The calculated average densities are in good agreement with measured average densities of MHSS samples $\left(\bar{\rho}=1.2 \mathrm{~g} / \mathrm{cm}^{3}\right)$. A second set of lattices (c.f. Table 2) comprises sequentially filled random distributions of circles with constant heights $h_{i}=0.58 \cdot R$. Consequently, these models exhibit constant radii $r \approx 0.815 \cdot R$ of the circular cross sections inside the cutting plane. The comparison of the lattices in Table 1 and 2 will show whether the effective thermal conductivity is dependent on particle radii $r$ or is mainly determined by the area fractions and distribution of spheres. Therefore, it is important that all meshes exhibit similar area fractions. 


\begin{tabular}{c|cccc}
\hline Lattice ID & $\begin{array}{c}A_{\mathrm{M}} \\
{[\%]}\end{array}$ & $\begin{array}{c}A_{\mathrm{S}} \\
{[\%]}\end{array}$ & $\begin{array}{c}A_{\mathrm{I}} \\
{[\%]}\end{array}$ & $\begin{array}{c}\text { Average density } \bar{\rho} \\
{\left[\mathrm{g} / \mathrm{cm}^{3}\right]}\end{array}$ \\
\hline$\# 1$ & 45.43 & 11.29 & 43.28 & 1.22 \\
$\# 2$ & 44.48 & 11.14 & 44.38 & 1.20 \\
$\# 3$ & 45.65 & 11.29 & 43.05 & 1.22 \\
$\# 4$ & 44.06 & 11.25 & 44.69 & 1.20 \\
$\# 5$ & 44.67 & 11.25 & 44.08 & 1.21 \\
$\# 6$ & 45.31 & 11.35 & 43.34 & 1.22 \\
$\# 7$ & 44.43 & 11.32 & 44.24 & 1.21 \\
$\# 8$ & 45.82 & 11.20 & 42.98 & 1.22 \\
$\# 9$ & 45.87 & 11.12 & 43.01 & 1.21 \\
$\# 10$ & 45.61 & 11.11 & 43.28 & 1.21 \\
$\# 11$ & 45.53 & 11.29 & 43.18 & 1.22 \\
$\# 12$ & 43.95 & 11.11 & 44.94 & 1.19 \\
\hline
\end{tabular}

Table 1 Area fractions and average density of Monte Carlo lattices ( $h_{i} \neq$ const.).

\begin{tabular}{c|cccc}
\hline Lattice ID & $\begin{array}{c}A_{\mathrm{M}} \\
{[\%]}\end{array}$ & $\begin{array}{c}A_{\mathrm{S}} \\
{[\%]}\end{array}$ & $\begin{array}{c}A_{\mathrm{I}} \\
{[\%]}\end{array}$ & $\begin{array}{c}\text { Average density } \bar{\rho} \\
{\left[\mathrm{g} / \mathrm{cm}^{3}\right]}\end{array}$ \\
\hline$\# 13$ & 44.68 & 11.19 & 44.13 & 1.20 \\
$\# 14$ & 44.62 & 11.19 & 44.19 & 1.20 \\
$\# 15$ & 44.68 & 11.20 & 44.12 & 1.20 \\
$\# 16$ & 44.67 & 11.19 & 44.14 & 1.20 \\
\hline
\end{tabular}

Table 2 Area fractions of lattices $\left(h_{i}=0.58 \cdot \mathrm{R}\right)$.

It is important to note that the analysis of two-dimensional cutting planes of three-dimensional MHSS introduces a major simplification. Only conduction inside the cutting plane can be simulated, but out-of-plane thermal conduction, i.e. along the curved surface of the metallic shells is neglected.

\section{Lattice Monte Carlo Method}

Thermal diffusion and atomic diffusion are both random processes that can be represented by random walks. The Einstein equation describes the particle diffusivity $D$ in $2 \mathrm{D}$ in the long-time limit:

$$
D=\frac{<\mathbf{R}^{2}>}{4 \cdot t}
$$

(where $\mathbf{R}$ is the vector displacement of a particle in time $t$ and the Dirac brackets refer to many particle histories) applies to both the thermal diffusivity $K$ and the self particle diffusivity when isolated heat entities and particles are considered. The thermal conductivity $\lambda_{\mathrm{i}}$ in a phase $i$ is related to the thermal diffusivity $K_{\mathrm{i}}$ in that phase by the expression $K_{\mathrm{i}}=\lambda_{i} / \rho_{\mathrm{i}} C_{\mathrm{p}}^{\mathrm{i}}$ where $\rho_{i}$ is the density of phase $i$ and $C_{\mathrm{p}}^{\mathrm{i}}$ is the specific heat of phase $i$. It is then possible to make use of the Einstein Equation in a Monte Carlo simulation for calculating the relative effective thermal conductivity $\lambda_{\text {eff }} \lambda_{i}\left(\lambda_{i}\right.$ is, for convenience, the maximum thermal conductivity of the individual phases present) by simply assigning the densities and the specific heats to be equal to unity in all phases. Then $\lambda_{\text {eff }} / \lambda_{i}$ equals the relative effective thermal diffusivity $K_{\text {eff }} / K_{i}$. Particles are released, one at a time, from randomly chosen sites within the lattice and each is permitted to explore the lattice on a random walk for the same time $t$. A time step is an attempt for the particle to jump at a given site. The conductivities (strictly diffusivities) of each phase are represented by different jump frequencies $\Gamma$ (strictly jump probabilities) noting that the diffusion coefficient can be expanded from Eq. 4 as (in 2D):

$$
D=\Gamma s^{2} / 4
$$


where $s$ is the jump distance in the lattice. The highest jump frequency is scaled to unity for efficiency. According to the thermal conductivities of the matrix $\lambda_{\mathrm{M}}=0.0214 \mathrm{~W} /(\mathrm{m} \cdot \mathrm{K})[14]$, the shell $\lambda_{\mathrm{S}}=51.9 \mathrm{~W} /(\mathrm{m} \cdot \mathrm{K})[18]$ and the inclusion $\lambda_{\mathrm{I}}=0 \mathrm{~W} /(\mathrm{m} \cdot \mathrm{K})$, the frequencies are $\Gamma_{\mathrm{M}}=0.00041$, $\Gamma_{\mathrm{S}}=1$ and $\Gamma_{\mathrm{I}}=0$.

\section{Results and discussion}

The effective thermal conductivities $\lambda_{\text {eff }}$ are determined using the Einstein Equation (Eq. 3). Table 3 shows the effective thermal conductivities $\lambda_{\text {eff }}$ of the Lattice Monte Carlo models with varying radii $r$ ( $h \neq$ const. ). The average value $\lambda_{\text {eff }}=1.00 \mathrm{~W} /(\mathrm{m} \cdot \mathrm{K})$ of the results is in excellent agreement with experimental findings $\left(\lambda_{\text {eff }}=1.01 \mathrm{~W} /(\mathrm{m} \cdot \mathrm{K})[14]\right)$. Despite the randomly distributed circular cross sections of spheres, the standard deviation of $\lambda_{\text {eff }}$ is only $0.015 \mathrm{~W} /(\mathrm{m} \cdot \mathrm{K})$ which corresponds to circa $1.5 \%$ of the average value. Preliminary investigations with a smaller amount of circular cross sections $(\approx 30)$ showed a higher standard deviation of $3.84 \%$.

\begin{tabular}{|c|c|c|c|c|c|c|c|c|c|c|c|c|}
\hline ID & $\# 1$ & \#2 & \#3 & \#4 & \#5 & \#6 & \#7 & \#8 & $\# 9$ & $\# 10$ & \#11 & $\# 12$ \\
\hline $\mathrm{W} /(\mathrm{m} \cdot \mathrm{K})]$ & 0.99 & 1.02 & 1.00 & 1.00 & 1.03 & 1.02 & 0.99 & 0.98 & 0.98 & 1.03 & 0.99 & 1.00 \\
\hline
\end{tabular}

Table 3 Effective thermal conductivity of the $2 D$ MHSS models ( $h_{i} \neq$ const.).

Table 4 shows the effective thermal conductivities of the lattice models with constant radii $r$ ( $h=$ const.). The average value of $\lambda_{\text {eff }}=1.01$ indicates no systematic dependence of the thermal conductivity on the distribution of the radii $r$ in the cutting plane.

\begin{tabular}{|c|c|c|c|c|}
\hline ID & $\# 13$ & $\# 14$ & $\# 15$ & $\# 16$ \\
\hline$\lambda_{\text {eff }} \quad[\mathrm{W} /(\mathrm{m} \cdot \mathrm{K})]$ & 1.02 & 1.02 & 0.99 & 1.00 \\
\hline
\end{tabular}

Table 4 Effective thermal conductivity of the $2 D$ MHSS models $(h=0.58 \cdot R)$.

Next, the effective thermal conductivity of a primitive cubic arrangement $(h=0.58 \cdot R)$ of circles is investigated. The distance between the spheres is chosen so that the structure exhibits similar area fractions as the lattices in Tables 2 and $3\left(A_{\mathrm{M}}=44.67 \%, A_{\mathrm{S}}=11.18 \%, A_{\mathrm{I}}=44.15 \%\right)$. A distinctly lower effective thermal conductivity $\lambda_{\mathrm{eff}}=0.70 \mathrm{~W} /(\mathrm{m} \cdot \mathrm{K})$ is now found. A likely explanation for this deviation is the regular arrangement of spheres which yields the maximum distance between two neighbouring spheres. Each sphere is surrounded by the low thermal conducting adhesive matrix which acts as a thermal insulator between the metallic shells.

To investigate the effect of substitution of $3 \mathrm{D}$ process by an appropriate $2 \mathrm{D}$ one we can invoke a Maxwell-type analytical solution for the effective conductivity [19]:

with

$$
\lambda_{\mathrm{eff}}=\frac{\lambda_{\mathrm{M}}\left(1-\frac{(d-1)\left(A_{\mathrm{I}}+A_{\mathrm{S}}\right)\left(\lambda_{\mathrm{M}}-\lambda_{\mathrm{SI}}\right)}{(d-1) \lambda_{\mathrm{M}}+\lambda_{\mathrm{SI}}}\right)}{1+\frac{\left(A_{\mathrm{I}}+A_{\mathrm{S}}\right)\left(\lambda_{\mathrm{M}}-\lambda_{\mathrm{SI}}\right)}{(d-1) \lambda_{\mathrm{M}}+\lambda_{\mathrm{SI}}}}
$$

$$
\lambda_{\mathrm{SI}}=\frac{\lambda_{\mathrm{S}}\left(1-\frac{(d-1) A_{\mathrm{I}}\left(\lambda_{\mathrm{S}}-\lambda_{\mathrm{I}}\right)}{\left(A_{\mathrm{I}}+A_{\mathrm{S}}\right)\left((d-1) \lambda_{\mathrm{S}}+\lambda_{\mathrm{I}}\right)}\right)}{1+\frac{A_{\mathrm{I}}\left(\lambda_{\mathrm{S}}-\lambda_{\mathrm{I}}\right)}{\left(A_{\mathrm{I}}+A_{\mathrm{S}}\right)\left((d-1) \lambda_{\mathrm{S}}+\lambda_{\mathrm{I}}\right)}}
$$

For the set of parameters used throughout this paper: $\left(A_{\mathrm{M}}=44.67 \%, A_{\mathrm{S}}=11.18 \%, A_{\mathrm{I}}=44.15 \%\right)$ and $\left(\lambda_{\mathrm{M}}=0.0214 \mathrm{~W} /(\mathrm{m} \cdot \mathrm{K}), \lambda_{\mathrm{S}}=51.9 \mathrm{~W} /(\mathrm{m} \cdot \mathrm{K}) \lambda_{\mathrm{I}}=0 \mathrm{~W} /(\mathrm{m} \cdot \mathrm{K})\right.$,) we soon find that according to the $3 \mathrm{D}$ version of the analysis $\lambda_{\text {eff }}=0.11270 \mathrm{~W} /(\mathrm{m} \cdot \mathrm{K})$ and according to the $2 \mathrm{D}$ version of the analysis $\lambda_{\text {eff }}=0.08235 \mathrm{~W} /(\mathrm{m} \cdot \mathrm{K})$. We can see that for the given parameters 'lowering' the dimensionality should produce about $25 \%$ lower values of the effective conductivity. (This is consistent with the 
last example where it was found that $\lambda_{\text {eff }}=0.70 \mathrm{~W} /(\mathrm{m} \cdot \mathrm{K})$ for the $2 \mathrm{D}$ version of the problem.) We point out that these analytical values are much lower than the values obtained by MC simulations. This is probably because of the close proximity of the composition to the critical percolation point where the coating phase becomes a network that can produce long-range thermal diffusion. Additional study of the transition from $3 \mathrm{D}$ to $2 \mathrm{D}$ around the critical composition is required to investigate this further.

\section{Conclusions}

In this paper, a Lattice Monte Carlo method has been used to determine the effective thermal conductivity in two dimensional models of adhesively bonded metallic hollow sphere structures (MHSS). In contrast to earlier approaches, more realistic distributions of spheres without the simplification of cubic symmetric arrangements were considered in this study. For the Monte Carlo analyses, two-dimensional periodic lattices representing different cutting planes through MHSS were generated. Therefore, an algorithm was used which sequentially fills the lattice by adding cut spherical shells and inclusions in the matrix. Another focus of this work was the analysis of the influence of different geometric circle distributions on the effective thermal conductivity. The findings of the random arrangements were also compared to a regular primitive cubic arrangement and with a Maxwell-type approach.

\section{Acknowledgements}

We wish to thank the Australian Research Council for its support of this work. T. Fiedler wants to acknowledge the Portuguese Foundation of Science and Technology (FCT) for financial support.

\section{References}

[1] H. Degischer and B. Kriszt: Handbook of Cellular Metals (WILEY-VCH, 2002).

[2] U. Ramamurty and A. Paul: Acta Mater. Vol. 52 (2004), p. 869.

[3] E. Solórzano, M.A. Rodríguez-Pérez, J.A. Reglero and J.A. de Saja: J. Mater. Sci., accepted for publication and in press.

[4] O.B. Olurin, M. Arnold, C. Körner and R.F. Singer: Mater. Sci. Eng. A Vol. 328 (2002), p. 334.

[5] T.J. Lu, H.A. Stone and M.F. Ashby: Acta Mater. Vol. 46 (1998), p. 3619.

[6] C.Y. Zhao, T.J. Lua, H.P. Hodson and J.D. Jackson: Mater. Sci. Eng. A Vol. 367 (2004), p. 123.

[7] W. Lu, C.Y. Zhao and S.A. Tassou: Int. J. Heat Mass Tran. Vol. 49 (2006), p. 2751.

[8] K. Boomsma, D. Poulikakos and F. Zwick: Mech. Mater. Vol. 35 (2003), p. 1161.

[9] E.J. Cookson, D.E. Floyd and A.J. Shih: Int. J. Mech. Sci. Vol. 48 (2006), p. 1314.

[10] T.J. Lu and C. Chen: Acta Mater. Vol. 47 (1999), p. 1469.

[11] E. Baumeister, S. Klaeger and A. Kaldos: J. Mater. Process. Tech. Vol. 155-156 (2004), p. 1839.

[12] T. Fiedler, A. Öchsner: Mater. Sci. Forum Vol. 553 (2007), p. 39.

[13] T. Fiedler, A. Öchsner: Mater. Sci. Forum Vol. 553 (2007), p. 45.

[14] T. Fiedler, E. Solórzano and A. Öchsner: Mater. Lett., accepted for publication.

[15] I.V. Belova and G.E. Murch: Sol. St. Phen. Vol. 128 (2007), p. 1.

[16] M. Jaeckel: German Patent 3724156 (1987).

[17] A. Rousset, J. Bonino, Y. Blottiere and C. Rossignol: French Patent 870740 (1987)

[18] J.R. Davis: ASM Specialty Handbook - Carbon and Alloy Steels, (ASM International, 1996).

[19] I.V. Belova and G.E. Murch: J. Phys. Chem. Solids Vol. 66 (2005), p. 722. 
Diffusion in Solids and Liquids III

doi:10.4028/www.scientific.net/DDF.273-276

Calculations of the Effective Thermal Conductivity in a Model of Syntactic Metallic Hollow Sphere Structures Using a Lattice Monte Carlo Method doi:10.4028/www.scientific.net/DDF.273-276.216 